\title{
Heat Shock Protein 70 (HSP70) Protein-Protein Interactions and a Putative Mechanism for the Potential Benefits of Heat Therapy for Type 2 Diabetes Mellitus
}

\author{
Magellan Guewo-Fokeng ${ }^{1,2}$, Eugene Sobngwi ${ }^{2,3,4}$, Barbara Atogho-Tiedeu ${ }^{2,5}$, \\ Jean-Claude Mbanya ${ }^{2,3,4}$, Wilfred Mbacham ${ }^{1,2, \text {, }}$ \\ ${ }^{1}$ Department of Biochemistry, Faculty of Medicine and Biomedical Sciences, University of Yaounde I, Yaounde, Cameroon \\ ${ }^{2}$ The Biotechnology Centre, University of Yaounde I, Nkolbisson, Yaounde, Cameroon \\ ${ }^{3}$ Department of Internal Medicine and Specialties, Faculty of Medicine and Biomedical Sciences, University of Yaounde I, Yaounde, Cameroon \\ ${ }^{4}$ National Obesity Centre, Diabetes, Endocrinology and Metabolic Diseases, Yaounde Central Hospital, Yaounde, Cameroon \\ ${ }^{5}$ Department of Biochemistry, Faculty of Science, University of Yaounde I, Yaounde, Cameroon
}

Email address:

guewomagellan@yahoo.fr (M. Guewo-Fokeng),wfmbacham@yahoo.com (W. Mbacham)

${ }^{*}$ Corresponding author

\section{To cite this article:}

Magellan Guewo-Fokeng, Eugene Sobngwi, Barbara Atogho-Tiedeu, Jean-Claude Mbanya, Wilfred Mbacham. Heat Shock Protein 70 (HSP70) Protein-Protein Interactions and a Putative Mechanism for the Potential Benefits of Heat Therapy for Type 2 Diabetes Mellitus. Biochemistry and Molecular Biology. Vol. 6, No. 2, 2021, pp. 19-24. doi: 10.11648/j.bmb.20210602.11

Received: March 4, 2021; Accepted: April 30, 2021; Published: May 8, 2021

\begin{abstract}
The use of heat therapy to treat diseases was very common in Africa. To date, the number of people using this form of therapy is very much on the decrease. Data on the molecular action mechanisms of how it might induce beneficial effects remain unknown. The aim of the present study was to make a contribution towards understanding the putative implication of Heat Shock Protein 70 (HSP70) in the pathophysiology of Type 2 Diabetes Mellitus (T2DM) and the hypothetical benefits of heat therapy through the establishment of a network of protein-protein interactions between Krüppel Like Factor 14 (KLF14), Transcription Factor 7 Like 2 (TCF7L2), Peroxisome Proliferator-Activated Receptor Gamma (PPARG) and HSP70 (HSPA4). Data were generated by the Search Tool for the Retrieval of Interacting Genes/Proteins (STRING) software version 10.0. This was used to identify the known and predicted protein-protein interactions (including direct or physical and indirect or functional associations) in the KLF14, TCF7L2, PPARG and HSP70 protein networks. With the active prediction methods (Gene Fusion, Neighborhood, Co-occurrence, Co-expression, Experiments, Databases and Text Mining) as interaction sources, Medium Confidence (0.400) and maximum number of interactions were used to show no more than 50 at the first shell and none at the second shell parameters. Fifty (50) proteins were identified to interact with these four proteins, namely KLF14, TCF7L2, PPARG and HSP70, resulting in a network diagram with 54 nodes (gene/proteins) and 485 edges, representing protein-protein associations. The network showed that HSP70 strongly interacts with other heat shock proteins like HSP90. The HSPBP, a cytoplasmic co-chaperone 1, inhibits HSPA1A chaperone activity by changing the conformation of its ATP-binding domain, thus interfering with this function. Based on the data generated by this in silico study, the potential beneficial effects of heat therapy on T2DM could probably be coordinated by the HSP70 protein-protein interactions involved in cell life and in the susceptibility to T2DM.
\end{abstract}

Keywords: Heat Therapy, Type 2 Diabetes Mellitus, Protein-Protein Interactions

\section{Introduction}

Type 2 Diabetes mellitus (T2DM) is a metabolic disorder characterized by chronic hyperglycemia and perturbations in protein, lipid and carbohydrate metabolism due to defects in insulin sensitivity and/or insulin resistance, which result in 
severe acute and chronic complications [1]. It is a complex disease that results from both environmental and genetic factors, with high morbidity and mortality rates in Asia, Latin America and principally in the sub-Saharan Africa region [2]. Since 2007 when the original publication proved the association of Transcription Factor 7 Like 2 (TCF7L2) gene variants with T2DM, this has been consistently replicated and confirmed in several ethnic groups [3, 4]. By Genome-Wide Association Studies and systematic large-scale surveys on associations between common DNA sequence variants and the disease, more than 67 (sixty-seven) genomic locations of proven signals associated to T2DM [5, 6] have been found, among which are TCF7L2 and PPARG, with major implications in insulin secretion and resistance [7, 8]. Recently, KLF14, a master gene for T2DM and obesity, was identified, driven by rs4731702 polymorphism [9].

In sub-Saharan Africa, the emerging co-occurrence of T2DM and tropical infectious diseases (communicable and non-communicable diseases) may have substantial implications, and the study by Danquah and collaborators showed that T2DM may actually increase the risk for malaria infection [10]. As stated by the International Diabetes Federation (IDF), T2DM is a worldwide health crisis with more than 463 million persons suffering from the disease, and this figure is projected to rise to 700 million by 2045 [2]. In the same line, the World Health Organization (WHO) noted that the global number of malaria cases fell from an estimated 262 million in 2000 to 229 million in 2019, though still high [11]. This drop could be attributed to the use of Intermittent Preventive Treatment (IPT) by pregnant women, a great variety of Artemisinin-based Combination Therapies (ACTs) and other drugs, and lastly, vector control strategies such as the use of long lasting Insecticide-Treated Nets (ITNs) and indoor residual sprays [12].

However, the number of people living in these endemic areas and using heat therapy to treat diseases is very much on the decrease. It was very common practice in Africa to treat diseases by this form of therapy. With the advent of new technologies (such as hot tubs and saunas), the data provided by researchers on this shows that it is becoming more and more sophisticated. Several studies have shown their benefits on people with certain metabolic disorders such as cardiovascular diseases $[13,14]$. A striking reduction of $1 \%$ in glycated hemoglobin (HbA1c) was observed in T2DM cases, suggesting this therapy diabetes management. The available studies on heat therapy show that in response to elevated temperature, a number of specific heat shock proteins (HSP) were identified to be upregulated in different types of organisms, including humans $[13,14]$. In addition to the fact that these HSPs are low in patients with T1DM (Type 1 Diabetes mellitus) and T2DM [15], they are involved in the prevention of apoptosis, the reparation of ion channels, and the protection of lipids, nucleic acids and proteins from damage by reducing oxidation [16]. Due to the small number of investigations in humans on heat therapy and its limitations, the molecular action mechanisms of how it induces these benefits remain unknown. This is why this study was undertaken to make a contribution to understanding the putative beneficial effect of Heat Shock Protein 70 (HSP70) in the pathophysiology of T2DM through the protein-protein interactions between KLF14, TCF7L2, PPARG and HSP70 (HSPA4).

\section{Materials and Methods}

\subsection{Data Sources}

A consortium made up of the Center for Protein Research (CPR) of Novo Nordisk Foundation, the European Molecular Biology Laboratory (EMBL), the University of Copenhagen (UCPH), The Swiss Institute of Bioinformatics (SIB), the Dresden University of Technology (DUT) and The University of Zurich (UZH) developed the STRING (Search Tool for the Retrieval of Interacting Genes/Proteins) software version 10.0 tool which was used to identify the known and predicted protein-protein interactions of the KLF14, TCF7L2, PPARG and HSP70 protein network. STRING is a comprehensive and authoritative database with information from many sources, including experimental data, computational prediction methods and public text collections. It currently covers more than 9,643,763 proteins from 2031 organisms and focuses mainly on their predicted and known interactions which include direct (physical) and indirect (functional) associations.

\subsection{Construction of Protein-Protein Interaction Network}

The protein-protein interactions of the KLF14, TCF7L2, PPARG and HSP70 protein network were built with STRING software version 10.0 under the active prediction methods (Gene Fusion, Neighborhood, Co-occurrence, Co-expression, Experiments, Databases and Text Mining) as interaction sources, with Medium Confidence (0.400) and maximum number of interactions were used to show no more than 50 at the first shell and none at the second shell parameters.

\section{Results and Discussion}

\subsection{Protein-Protein Interactions Network}

Fifty proteins (CTNNB1, STIP1, PPARGC1A, MED1, NCOA1, EP300, HSP90AA1, HIF1A, STUB1, UBC, BAG1, NCOR1, DNAJB1, NCOA2, HDAC3, BAG3, TP53, LEP, ADIPOQ, CTBP1, NCOR2, GRPEL2, SLC2A4, SNCA, GRPEL1, CD36, NCOA6, KLF4, NCOA3, PARK2, ESR1, CREBBP, TLE1, PLIN1, TGFB1, AXIN2, TLE4, JUP, GCG, TLE2, NLK, PCK1, IL4, SP1, CLPB, SUMO1, HSPBP1, T, IL2, DKK1) were identified to interact with these four proteins (KLF14, TCF7L2, PPARG and HSP70). This resulted in a network diagram with 54 nodes (gene/protein - including the KLF14, TCF7L2, PPARG and HSP70 ones) and 485 edges (representing protein-protein associations) or interactions (Figure 1). This diagram was built from two proteins/genes mainly implicated in T2DM and obesity, one master gene for T2DM and obesity, and HSP70. This last one is a member of the HSP family that has the capacity to protect cell proteins from 
denaturation or damage induced by various stressful stimuli. It does so by binding to denatured or inappropriately folded proteins [17]. Heat shock treatment of mice has been shown to result in strong induction of HSP70 in multiple organs and to confer significant protection against tumor necrosis factor
(TNF)-induced lethality. [18]. In 2003, Zouari and collaborators carried out a study on obese Tunisians which suggested that HSP70-2 polymorphism has susceptibility implications in both obesity and T2DM [19].

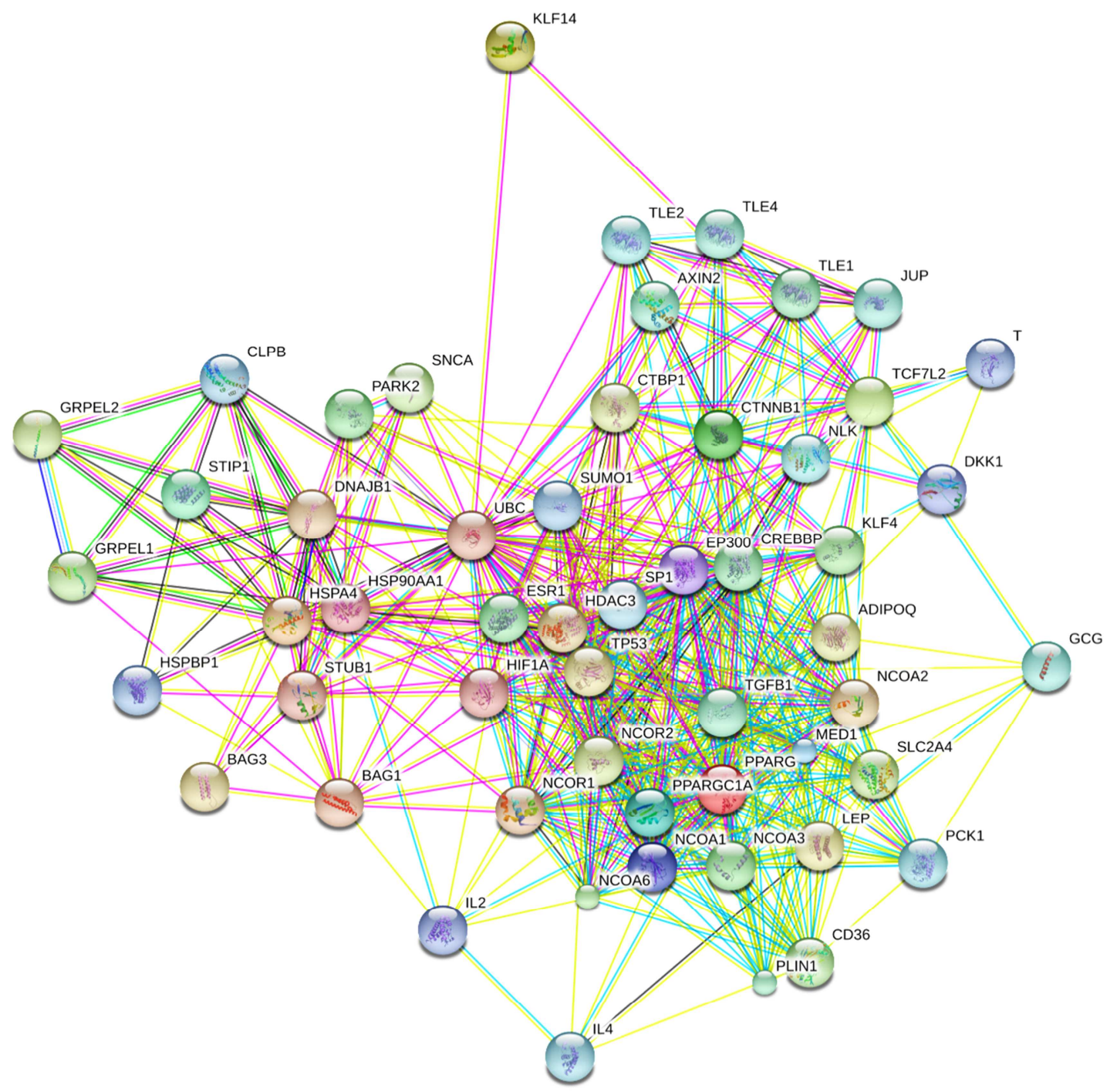

Figure 1. Network protein-protein interactions of KLF14, TCF7L2, PPARG and HSP70/HSPA4 proteins.

\subsection{Interpretation of Protein-Protein Network}

This network generated by protein-protein interactions between KLF14, TCF7L2, PPARG and HSP70 proteins shows that the HSP70 (HSPA4 - a component of the ribosome-associated complex involved in folding or maintaining nascent polypeptides in a folding-competent state) strongly interacts with another heat shock protein, HSP90 (HSP90AA1, - a molecular chaperone that promotes the maturation, structural maintenance and proper regulation of specific target proteins involved for instance in cell cycle control and signal transduction). It also interacts with HSPBP1 (HSPA - a 70kDa heat shock binding protein), interacts with cytoplasmic co-chaperone 1, and inhibits HSPA1A chaperone activity (changes the conformation of the
ATP-binding domain of HSPA1A and interferes with ATP binding). It interferes with ubiquitination mediated by STUB1 (implicated in the collaboration of ATXN3 in the degradation of misfolded chaperone substrates-ATXN3 restricting the length of ubiquitin chain attached to STUB1/CHIP substrates and preventing further chain extension), Ubiquitination of NOS1 in concert with HSP70 and HSP40 which interacts with HSP70 and can stimulate the ATPase activity of DnaJ (HSP40) homolog, subfamily B, member 1 . All this shows that the expression of HSP70 induced by heat therapy may stimulate the co-expression of other HSPs (HSP40, HPS90).

On the other hand, the presence of many proteins/genes associated to T2DM is demonstrated in this network. These include EP300 which is an E1A-associated cellular p300 transcriptional co-activator protein that is implicated in 
transcription regulation via chromatin remodeling. It plays an important role in cell proliferation and differentiation processes and regulates cAMP-gene by binding specifically to the phosphorylated CREB protein. Transforming Growth Factor Beta 1 (TGFB1), also associated to T2DM and synthesized by many cells, is a protein that controls differentiation, proliferation, and other functions.

The $2509 \mathrm{C} / \mathrm{T}$ polymorphism of the gene that encodes this protein is shown to play an important role in T2DM by increasing diabetic retinopathy susceptibility [20]). ADIPOQ is located on human chromosome $3 \mathrm{q} 27$, and is identified as a predisposition locus for metabolic syndrome and T2DM [21]. Adiponectin, C1Q and collagen domain contain an important adipokine involved in the control of fat metabolism and insulin sensitivity, with direct anti-diabetic, anti-atherogenic and anti-inflammatory activities.

Peroxisome Proliferator-Activated Receptor Gamma, Coactivator 1 Alpha (PPARGC1A) is a co-activator that interacts with PPARG [22], and is associated to T2DM by the Thr394Thr polymorphism in Asian Indians [23] and by rs4235308 and rs7656250 polymorphisms between Haitian Americans and African Americans. Pointing out differences within the Black race [24]) shows that by the elevation of body temperature the co-expression of HSPs may influence the amount of these proteins whose gene variants are implicated in the susceptibility to T2DM. It is important to note the position of the KLF14 protein (as a master controller) in this network. This protein only interacts with TCF7L2 (strongly implicated in the predisposition to T2DM) and with UBC (suggesting their regulation by degradation via ubiquitination; previously found in the regulatory network of the KLF14 gene [25].

\section{Conclusion}

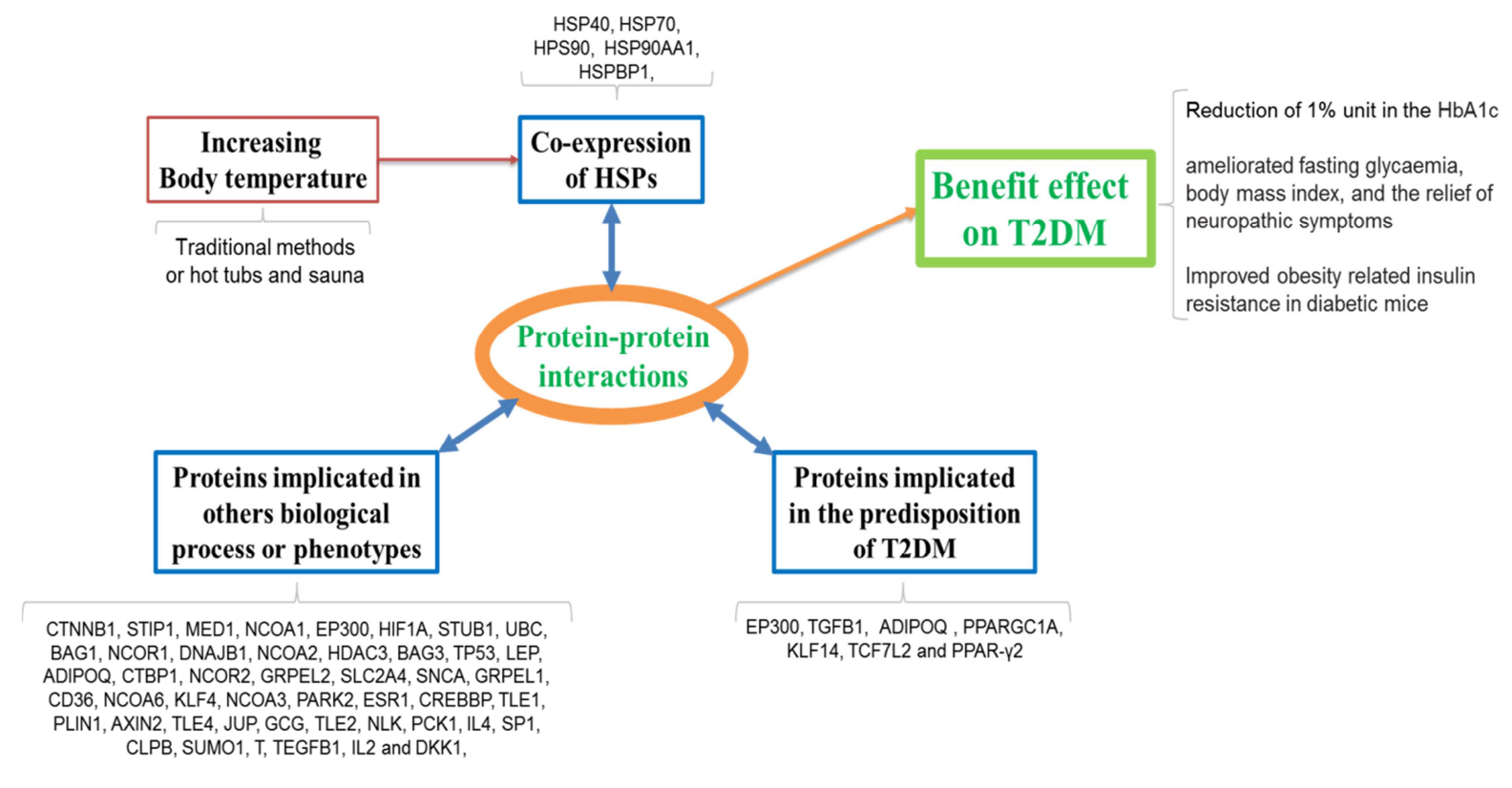

Figure 2. Hypothetic model describing the role of protein-protein interactions in the beneficial effect of heat therapy for T2DM.

Data generated by this in silico study shows that the

potential beneficial effects of heat therapy on T2DM could
Finally, UBC (a member of the Ubiquitin protein family, central node interacts with the majority of the proteins in this network. This protein is implicated in protein trafficking, endocytosis, kinase modification, cell cycle regulation, apoptosis, DNA repair, and regulation of other cell signaling pathways and also principally in protein degradation through the $26 \mathrm{~S}$ proteasome by ubiquitination [26]. It could therefore regulate the amount of these proteins, including KLF14, TCF7L2, PPARG and HSP70 and other proteins by their degradation.

\subsection{Possible Explanation of Heat Therapy in T2DM}

Three (3) weeks of heat therapy for T2DM patients by daily hot tub submersion were demonstrated by Hooper in 1999 to improve on fasting glycaemia, body mass index, and the relief of neuropathic symptoms [14]. This result was also demonstrated in animal models, where Kokura and collaborators showed that it was possible to improve obesity-related insulin resistance in diabetic mice by increasing body temperature, thus inducing the expression of HSPs [27].

From the network of protein-protein interactions generated by KLF14, TCF7L2, PPARG and HSP70 (Figure 1), in addition to studies by Kurucz, Hooper, Kokura and collaborators $[14,15,27]$, we propose the explanation that increasing body temperature improves the co-expression of Heat Shock Proteins (HSPs) at the cellular level. These proteins interact with a number of other proteins, including those encoded by genes involved in the predisposition to T2DM, to yield potential beneficial effects (Figure 2). 
probably be coordinated by the HSP70 protein-protein interactions involved in cell life and in the susceptibility to T2DM (KLF14, TCF7L2, PPARG, EP300, ADIPOQ, TGFB1 and PPARGC1A), certainly by a multi-regulatory action of the expression or the activity of these. This network of protein-protein interactions which helps to bring this understanding is just one part of the whole complex network that exists in the cell. Further investigations using an appropriate sample size are required to validate these first data.

\section{Abbreviations}

KLF14 : Kruppel-like factor 14 ; TCF7L2 : transcription factor 7-like 2 ; PPARG : peroxisome ; proliferator-activated receptor gamma ; HSP27 : HSPB 1 - heat shock $27 \mathrm{kDa}$ protein 1 ; HSP47 : SERPINH1 - serpin peptidase inhibitor, clade $H$ (heat shock protein 47 ), member 1, (collagen binding protein 1) ; HSP60 : HSPD1 - heat shock $60 \mathrm{kDa}$ protein 1 (chaperonin) ; HSPA4 : heat shock $70 \mathrm{kDa}$ protein 4 ; HSP90 : HSP90AA1 - heat shock protein 90kDa alpha (cytosolic), class A member 1 ; HSP110 : HSPH1 - heat shock $105 \mathrm{kDa} / 110 \mathrm{kDa}$ protein 1 ; CTNNB1 : Catenin (cadherin-associated protein), beta 1 ; STIP 1 : stress-induced-phosphoprotein 1 ; PPARGC1A : Peroxisome proliferator-activated receptor gamma, coactivator 1 alpha ; MED1 : Mediator complex subunit 1 ; NCOA1 : nuclear receptor coactivator 1 ; EP300 : E1A binding protein p300; HSP90AA1 : heat shock protein $90 \mathrm{kDa}$ alpha (cytosolic), class A member 1; HIF1A : Hypoxia inducible factor 1, alpha subunit ; STUB1 : STIP1 homology and U-box containing protein 1, E3 ubiquitin protein ligase; UBC : ubiquitin C ; BAG1 : BCL2-associated athanogene ; NCOR1 : nuclear receptor corepressor 1 ; DNAJB1 : DnaJ (Hsp40) homolog, subfamily $\mathrm{B}$, member 1 ; NCOA2 : nuclear receptor coactivator 2 ; HDAC3 : histone deacetylase 3 ; BAG3 : BCL2-associated athanogene 3; TP53 : tumor protein $\mathrm{p} 53$; LEP : leptin ; ADIPOQ : adiponectin, C1Q and collagen domain containing ; CTBP 1 : C-terminal binding protein 1 ; NCOR2 : nuclear receptor corepressor 2 ; GRPEL : GrpE-like 2, mitochondrial (E.coli) ; SLC2A4 : solute carrier family 2 (facilitated glucose transporter), member 4 ; SNCA : synuclein, alpha (non A4 component of amyloid precursor) ; CD36 : CD36 molecule (thrombospondin receptor) ; NCOA6 : nuclear receptor coactivator 6 ; KLF4 : Kruppel-like factor 4 (gut) ; NCOA3 : nuclear receptor coactivator 3 ; PARK2 : parkinson protein 2, E3 ubiquitin protein ligase (parkin) ; ESR1 : estrogen receptor 1 ; CREBBP : CREB binding protein ; TLE1 : transducin-like enhancer of split 1 (E(sp1) ; PLIN1 : perilipin 1 ; TGFB1 : transforming growth factor, beta 1 ; AXIN2 : axin 2 ; TLE4 : transducin-like enhancer of split 4 (E(sp1) ; JUP : junction plakoglobin ; GCG : glucagon ; TLE2 : transducin-like enhancer of split 2 (E(sp1) ; NLK : nemo-like kinase ; PCK1 : phosphoenolpyruvate carboxykinase 1 (soluble) ; IL4 : interleukin 4 ; SP1 : Sp1 transcription factor ; CLPB : ClpB caseinolytic peptidase B ; SUMO1 : SMT3 suppressor of mif two 3 homolog 1 (S. cerevisiae) ; HSPBP1 : HSPA (heat shock $70 \mathrm{kDa}$ ) binding protein, cytoplasmic cochaperone 1 ; $\mathrm{T}$ : T, brachyury homolog ; IL2 : Interleukin 2 ; DKK1 : dickkopf 1 homolog.

\section{Author Contribution}

Conception and design of experiments: Magellan Guewo-Fokeng, Eugene Sobngwi and Wilfred Mbacham. Data collection and analysis: Magellan Guewo-Fokeng. Data interpretation: Magellan Guewo-Fokeng, Eugene Sobngwi, Barbara Atogho-Tiedeu, Jean-Claude Mbanya and Wilfred Mbacham. Preparation of the first draft of the manuscript: Magellan Guewo-Fokeng. Critical discussion and manuscript revision: Magellan Guewo-Fokeng, Eugene Sobngwi, Barbara Atogho-Tiedeu, Jean-Claude Mbanya and Wilfred Mbacham. Final editing and approval of the manuscript: Eugene Sobngwi, Barbara Atogho-Tiedeu, Jean-Claude Mbanya and Wilfred Mbacham. All the authors read and approved the final version of the manuscript.

\section{Disclosure Statement}

The authors declare that they have no competing interests.

\section{Acknowledgements}

The authors greatly thank the Laboratory for Public Health Research Biotechnology (LAPHER-BIOTECH) and the Laboratory for Molecular Medicine and Metabolism (LMMM) of the Biotechnology Center of the University of Yaoundé I. This work received pre-funding from Professor Eugene Sobngwi and Professor Wilfred Mbacham pending funding from the African Partnership for Chronic Disease Research strategic award from the UK Medical Research Council under the RC/DFID Concordat agreement (Grant Number MR/K013491/1).

\section{References}

[1] Guillausseau PJ, Meas T, Virally M, Laloi-Michelin M, Médeau V, Kevorkian JP. (2008). Abnormalities in insulin secretion in type 2 diabetes mellitus. Diabetes Metab, 34 Suppl 2: S43-S48.

[2] International Diabetes Federation: IDF Diabetes Atlas. 9th edition. 2019 Available at: http://www.idf.org/diabetesatlas. Accessed: January 05, 2021.

[3] Cauchi S. (2006). Transcription factor TCF7L2 genetic study in the French population: Expression in human beta-cells and adipose tissue and strong association with type 2 diabetes. Diabetes. 55: 2903-2908.

[4] Cauchi S. (2007). TCF7L2 is reproducibly associated with type 2 diabetes in various ethnic groups: a global meta-analysis. J. Mol. Med. 85: 777-782 
[5] Sladek R, Rocheleau G, Rung J, Dina C, Shen L, Serre D, Boutin P, Vincent D, Belisle A, Hadjadj S, Balkau B, Heude B, Charpentier G, Hudson TJ, Montpetit A, Pshezhetsky AV, Prentki M, Posner BI, Balding DJ, Meyre D, Polychronakos C, Froguel P. (2007). A genome-wide association study identifies novel risk loci for type 2 diabetes. Nature, 445: 881-885.

[6] McCarthy MI. (2010). Genomics, Type 2 Diabetes, and Obesity. New England Journal of Medicine. 363: 2339-2350

[7] Loss RJ, Franks PW, Francis RW, Barroso I, Gribble FM, Savage DB. (2007). TCF7L2 polymorphisms modulate proinsulin levels and beta-cell function in a British Europid population. Diabetes. 56: 1943-1947.

[8] Deeb SS, Fajas L, Nemoto M, Pihlajamaki J, Mykkanen L, Kuusisto J, Laakso M, Fujimoto W, Auwerx J. (1998). A Pro12Ala substitution in PPAR $\gamma 2$ associated with decreased receptor activity, lower body mass index and improved insulin sensitivity. Nat Genet. 20 (3): 284-287.

[9] Kerrin SS, Asa KH, Grundberg E, Nica AC, Thorleifsson G, Kong A, Thorsteindottir U, So-Youn S, Hannah BR, the GIANT Consortium, the MAGIC Investigators, the DIAGRAM Consortium, Soranzo N, Ahmadi KR, Lindgren CM, Stefansson K, Dermitzakis ET, Deloukas P, Spector TD, McCarthy MI. (2011). Identification of an imprinted master trans-regulator at the KLF14 locus related to multiple metabolic phenotypes. Nature Genetic. 43 (6): 561-564.

[10] Danquah I, Bedu-Addo G, and Mockenhaupt FP. (2010). Type 2 diabetes mellitus and increased risk for malaria infection. Emerging Infectious Diseases. 6 (10): 1601-1604.

[11] World Health Organization, "Trends in infection prevalence, case incidence and mortality rates" 2020.

[12] Steketee RW and Campbell CC. (2010). Impact of national malaria control scale-up programmes in Africa: Magnitude and attribution of effects. Malaria journal. 9 (1): 299.

[13] Crinnion WJ. (2011). Sauna as a valuable clinical tool for cardiovascular, autoimmune, toxicant-induced and other chronic health problems. Altern Med Rev. 16: 215-225.

[14] Hooper PL. (1999). Hot-tub therapy for type 2 diabetes mellitus. N Engl J Med. 341: 924-925.

[15] Kurucz I, Morva A, Vaag A, Eriksson KF, Huang X, Groop L, Koranyi L. (2002). Decreased expression of heat shock protein 72 in skeletal muscle of patients with type 2 diabetes correlates with insulin resistance. Diabetes. 51: 1102-1109

[16] Benjamin IJ, McMillan DR. (1998). Stress (heat shock) proteins: Molecular chaperones in cardiovascular biology and disease. Circ Res. 83: 117-132

[17] Van Buskirk AM, De Nagel DC, Gualiardi LE. (1991).
Cellular and subcellular distribution of PBP 72/74, a peptide-binding protein that plays a role in antigen processing. J Immunol. 146: 500-506.

[18] Van Molle W1, Wielockx B, Mahieu T, Takada M, Taniguchi T, Sekikawa K, Libert C. (2002). HSP70 protects against TNF-Induced inflammatory shock. Immunity. 16: 685-695

[19] Zouari Bouassida K, Chouchane L, Jellouli K, Chérif S, Haddad S, Gabbouj S, Danguir J. (2004). Polymorphism of stress protein HSP70-2 gene in Tunisians: Susceptibility implications in type 2 diabetes and obesity. Diabetes Metab. 30 (2): $175-180$

[20] Paine SK, Basu A, Mondal LK, Sen A, Choudhuri S, Chowdhury IH, Saha A, Bhadhuri G, Mukherjee A, Bhattacharya B. (2012). Association of vascular endothelial growth factor, transforming growth factor beta, and interferon gamma gene polymorphisms with proliferative diabetic retinopathy in patients with type 2 diabetes. Mol Vis. 18: $2749-2757$.

[21] Cox AJ, Lambird JE, An SS, Register TC, Langefeld CD, Carr JJ, Freedman BI, Bowden DW. (2013). Variants in adiponectin signaling pathway genes show little association with subclinical CVD in the diabetes heart study. Obesity. 21: E456-E462.

[22] Sears IB, MacGinnitie MA, Kovacs LG, Graves RA. (1996). Differentiation-dependent expression of the brown adipocyte uncoupling protein gene: Regulation by peroxisome proliferator-activated receptor gamma. Mol Cell Biol. 16: 3410-3419.

[23] Vimaleswaran KS, Radha V, Ghosh S, Majumder PP, Babu HNS, Rao MR, Mohan V. (2005). Peroxisome proliferator activated receptor gamma $\mathrm{C}$ coactivator-1 (PGC-1) gene polymorphisms and their relationship to type 2 diabetes in Asian Indians. Diabet Med. 22: 1516-1521.

[24] Cheema AK, Li T, Liuzzi JP, Zarini GG, Dorak MT, and Huffman FG. (2015). Genetic Associations of PPARGC1A with Type 2 Diabetes: Differences among populations with African Origins. Diabet Res. ID 921274, 10 pages

[25] Guewo-Fokeng M, Atogho-Tiedeu B, Sobngwi E, Mbanya JC, Mbacham W. (2016). The Krüppel-Like Factor 14 (KLF14) master gene of multiple metabolic phenotypes: Putative Trans-Regulator Network. J Transl Biomed. 7 (2):67.

[26] Clague MJ, and Urbé S. (2010). Ubiquitin: Same molecule, different degradation pathways. Cell. 143: 682-685.

[27] Kokura S, Adachi S, Manabe E, Mizushima K, Hattori T, Okuda T, Nakabe N, Handa O, Takagi T, Naito Y, Yosida N, Yoshikawa T. (2007). Whole body hyperthermia improves obesity-induced insulin resistance in diabetic mice. Int $\mathrm{J}$ Hypertherm. 23 (3): 259-265. 